\title{
ANALYSIS OF MULTIPATH PIXELS IN SAR IMAGES
}

\author{
J. W. Zhao ${ }^{\text {a,b }}$, J. C.Wu ${ }^{\text {a,* }}$, X. L. Ding ${ }^{\text {, }}$, L. Zhang ${ }^{b}$, F. M.Hu ${ }^{\text {a }}$ \\ ${ }^{a}$ College of Surveying and Geo-Informatics, Tongji University, 1239 Siping Road, Shanghai, China - mnls0226@ gmail.com, \\ jcwu@tongji.edu.cn, dogfish.hu@outlook.com \\ ${ }^{\mathrm{b}}$ Dept. of Land Surveying \& Geo-Informatics, The Hong Kong Polytechnic University, Hung Hom,Kowloon,Hong Kong - \\ mnls0226@gmail.com, (xl.ding, lslzhang)@polyu.edu.hk
}

\section{Theme Sessions, ThS 7}

KEY WORDS: Multipath, Signal Propagation, RaySAR, Statistic Index, Characteristics Of Radar Signal, Analysis Of Multipath Pixels

\begin{abstract}
:
As the received radar signal is the sum of signal contributions overlaid in one single pixel regardless of the travel path, the multipath effect should be seriously tackled as the multiple bounce returns are added to direct scatter echoes which leads to ghost scatters. Most of the existing solution towards the multipath is to recover the signal propagation path. To facilitate the signal propagation simulation process, plenty of aspects such as sensor parameters, the geometry of the objects (shape, location, orientation, mutual position between adjacent buildings) and the physical parameters of the surface (roughness, correlation length, permittivity)which determine the strength of radar signal backscattered to the SAR sensor should be given in previous. However, it's not practical to obtain the highly detailed object model in unfamiliar area by field survey as it's a laborious work and time-consuming. In this paper, SAR imaging simulation based on RaySAR is conducted at first aiming at basic understanding of multipath effects and for further comparison. Besides of the pre-imaging simulation, the product of the after-imaging, which refers to radar images is also taken into consideration. Both Cosmo-SkyMed ascending and descending SAR images of Lupu Bridge in Shanghai are used for the experiment. As a result, the reflectivity map and signal distribution map of different bounce level are simulated and validated by 3D real model. The statistic indexes such as the phase stability, mean amplitude, amplitude dispersion, coherence and mean-sigma ratio in case of layover are analyzed with combination of the RaySAR output.
\end{abstract}

\section{INTRODUCTION}

Multipath propagation is a tricky problem in SAR imaging processing. By now, few research on multipath have been done. Those pixels corresponding to the multipath incorporate false travel path leading to ghost scatters(Auer et al., 2011). Especially in high-rise urban area, the buildings are densely distributed, leading to much more complicated imaging environment. To recover the true signal travel path, namely, signal transmission from the sensor, interaction with illuminating objects on earth surface, sum of backscattering energy at receiver, several SAR image simulation approaches, such as RaySAR(Auer, 2011), CohRaS(Hammer and Schulz, 2009), and SARViz(Balz and Stilla, 2009), can be employed to reveal the relationship between signatures in SAR intensity images and building geometry. Except for the SAR simulation approach, SAR data itself can be divided into amplitude and phase, which carry valuable information about the radar signal and illuminating object.

As a whole, this paper aims at analyzing those pixels contaminated by the multipath propagation and distinguishing different bounce level. Both SAR image simulation and signal statistic analysis are employed.

\section{SCENE OF INTEREST AND DATA SET}

\subsection{Scene of interest}

Officially put into operation in 2003, Lupu Bridge(as shown in Figure 1) is the first all-steel arch bridge in Huangpu River in
Shanghai. What's more, it made several breakthroughs at that time, such as the second long span steel arch bridge in the world and the first fully welding connected huge arch bridge. There are sightseeing pavements on the two bridge arcs on both sides.

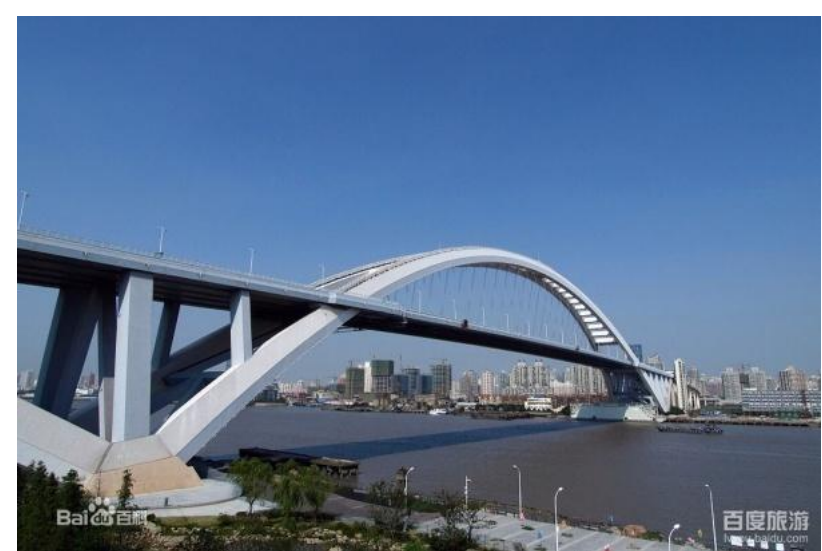

Figure 1.A perspective view onto the Lupu Bridge(@ Baidu Image Copyright 2016)

\subsection{Data set}

Both Cosmo-SkyMed ascending and descending SAR images of Lupu Bridge in Shanghai are used for the experiment and it is a coincidence that the span direction of the bridge is more or less parallel to the fly direction, the bridge is then an ideal choice for multipath analysis. The basic structure of the bridge is relatively obvious in the intensity images and the imaging environment is relatively simple which facilitate the analysis of different 
bounce level. Useful information about the two tracks can be found in Table 1. Preliminary data processing is done with GAMMA software.

\begin{tabular}{|l|c|c|}
\hline & Descending & Ascending \\
\hline Time Interval & $\begin{array}{c}20080518- \\
20100601\end{array}$ & $\begin{array}{c}20081210- \\
20101106\end{array}$ \\
\hline Number & 35 & 35 \\
\hline Azimuth(Line) & 450 & 400 \\
\hline Range(Column) & 320 & 250 \\
\hline Incidence Angle(Deg) & 40 & 40 \\
\hline Heading(Deg) & -168.76 & -10.34 \\
\hline $\begin{array}{l}\text { Azimuth } \\
\text { Resolution(M) }\end{array}$ & 1.8 & 2.25 \\
\hline Range Resolution(M) & 0.97 & 1.25 \\
\hline
\end{tabular}

Table 1. Useful information about the two track Cosmo-SkyMed SAR images

\section{SAR IMAGE SIMULATION}

SAR image simulation is conducted with RaySAR, a three dimensional SAR imaging simulation software based on Pov-Ray (a free, open-source and three-dimensional image rendering software using ray tracing method). RaySAR was developed by Dr. Stefan in Technical University Munich, Germany and turned into open-resource in early 2016. With assumed reflection model, known 3D building model, satellite parameters and physical information of the surface material, SAR reflectivity maps can be simulated. Backward from the image plane, signal contributions of certain pixel can be relocated in the real 3D world, which facilitates a better understanding of the generation and effects of multipath.

\subsection{Preparation of data}

The 3D bridge model(as shown in Figure 2) is obtained from Google 3D Warehouse. The basic structure(two arcs, road, pillars under bridge on both sides, horizontal bars in the centre and vertical bars on both sides, tower on both sides, railing of the road and arc, seil and triangular framework at bottom between two arcs) of the bridge is well represented and the size, location and orientation are basically accurate. Later on, the model should be transformed into Pov-Ray format and parameters of the sensor, radar geometry and object surface attributes should be set in previous.

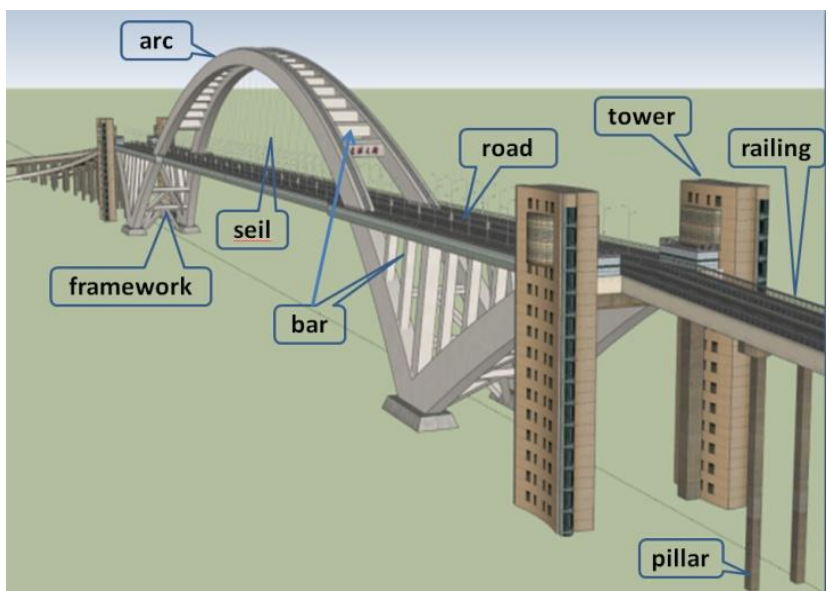

Figure 2. 3D model of the Lupu Bridge (CGoogle 3D Warehouse)

\subsection{Simulated reflectivity maps}

A flat surface is defined beneath the bridge to represent the water surface. Specular and diffuse reflection of the radar signal is enabled. After ray tracing, reflectivity maps of both tracks(Figure 3) are provided whose sampling is adapted to the real SAR data. For comparison, averaged SAR intensity images are shown in Figure 4. Clipping threshold 0.4 was chosen to better visualize RaySAR reflectivity maps.

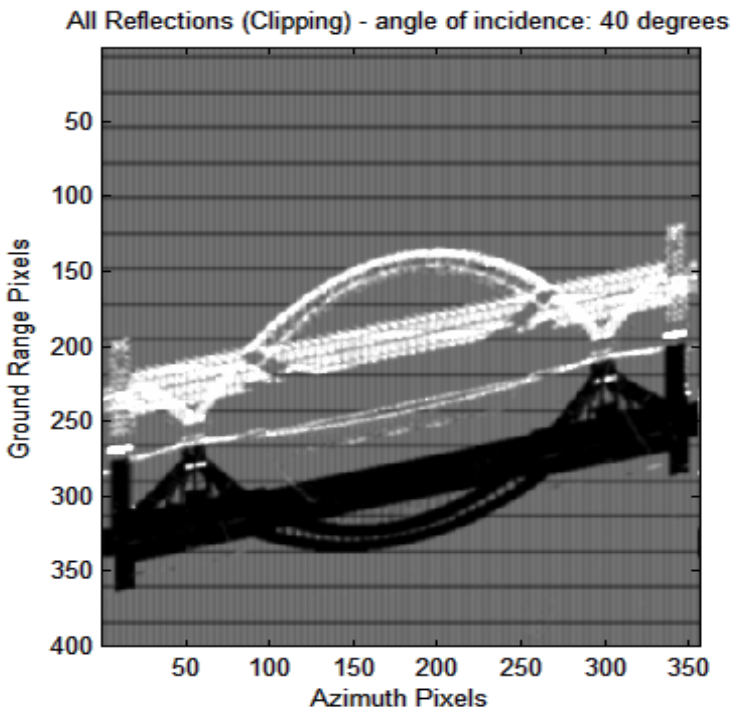

(a)

All Reflections (Clipping) - angle of incidence: $\mathbf{4 0}$ degrees

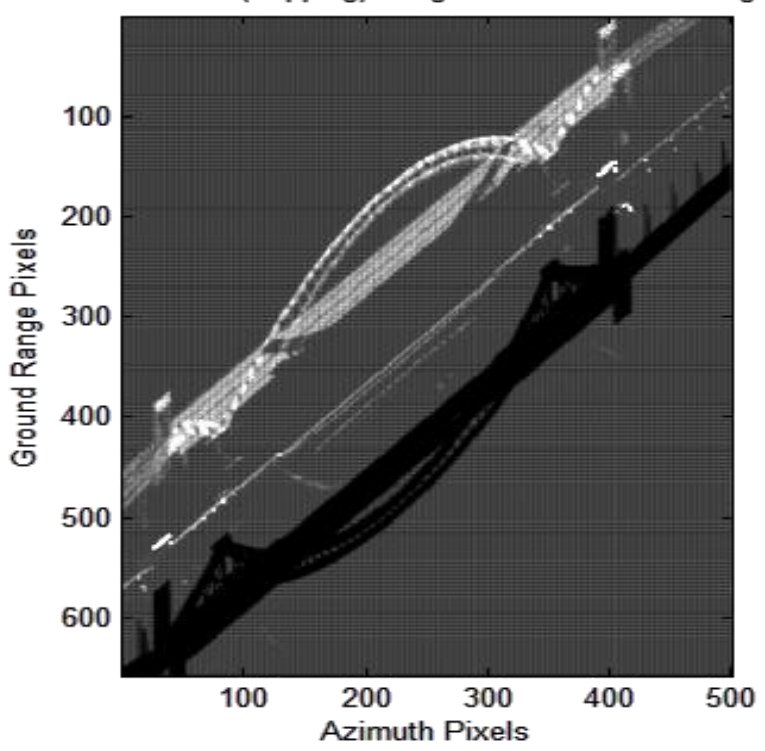

(b)

Figure 3.RaySAR simulated reflectivity maps (a) Ascending track (b) Descending track 


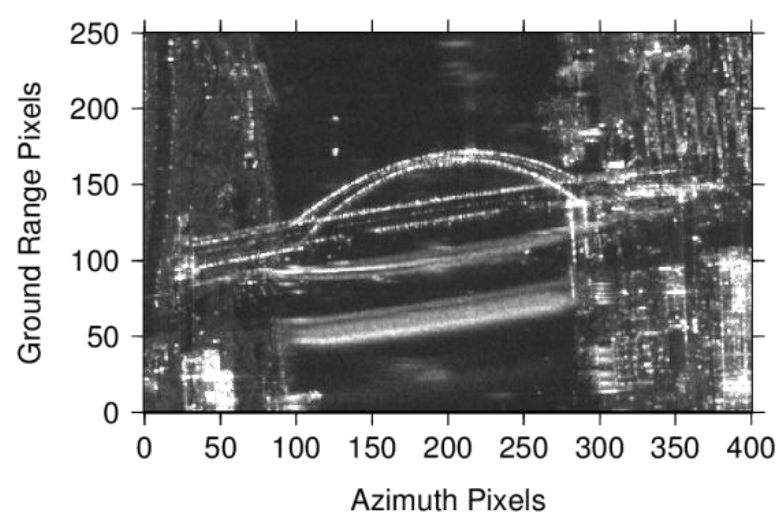

(a)

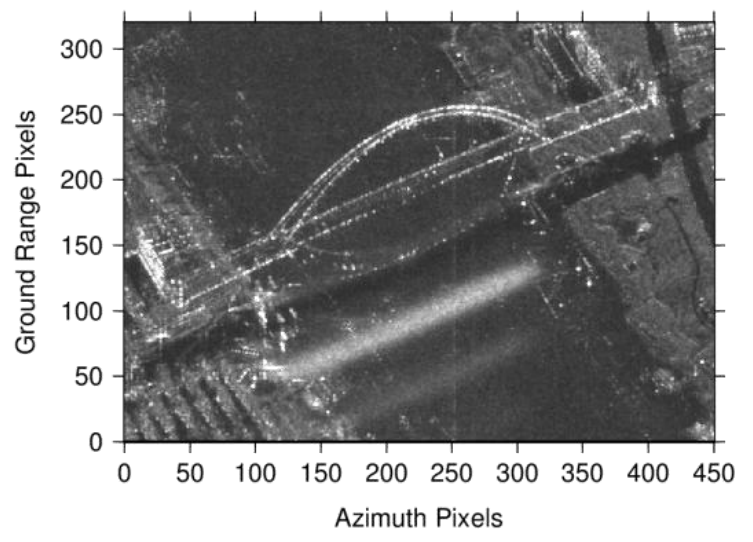

(b)

Figure 4. Averaged SAR intensity image (a) Ascending track (b) Descending track

Note the coordinate system, size and absolute intensity between RaySAR reflectivity maps and averaged SAR intensity images are different. The relative shift between RaySAR reflectivity maps and SAR averaged intensity images can be determined by cross-correlating the reflectivity map with the SAR image section in frequency domain. This will be done in future work. Simply seen from the comparison between Figure 3 and 4, the pattern of prominent signal such as two bridge arcs, the reflection of arc and road in the water and the shadow of the bridge, are identical. During the ray-tracing process, the water surface is treated as an ideal reflector. The arc and bar have relative higher reflection and diffusion as they are covered with steel. The pillar and seil have relatively lower reflection and diffusion as there surface are much more rough. Nevertheless, some difference inevitably exists. The most obvious difference is the overestimation of the frameworks and road surface. Besides. the towers on both sides should have a variety of signal pattern due to the incidence angular dependence of diffusion. The main reason is expected to be the limited level of detail of the bridge model as, for instance, the facades and roofs of the towers on both sides lack geometrical description.

\subsection{Simulated signal contribution}

Thanks to the ray-tracing method, the simulated SAR signals can be decomposed into different bounce levels and there 3D distribution can be shown in both image coordinate and bridge model coordinate. Figure 5 shows the simulated signal distribution of both tracks.

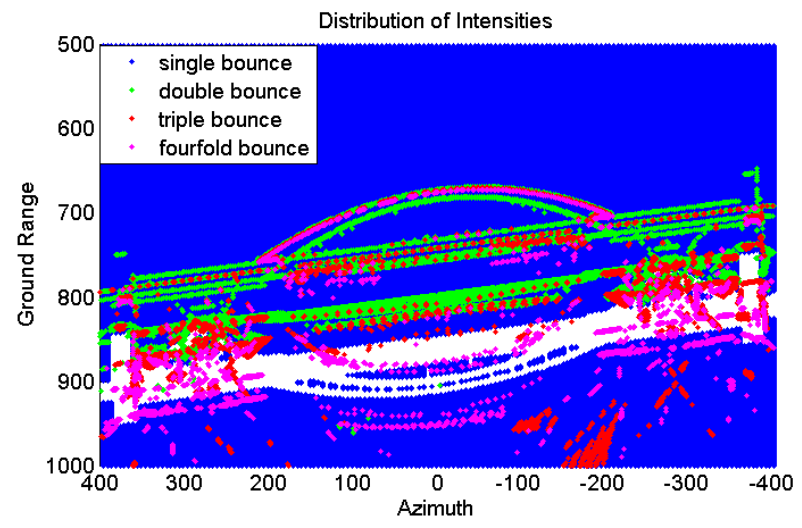

(a)

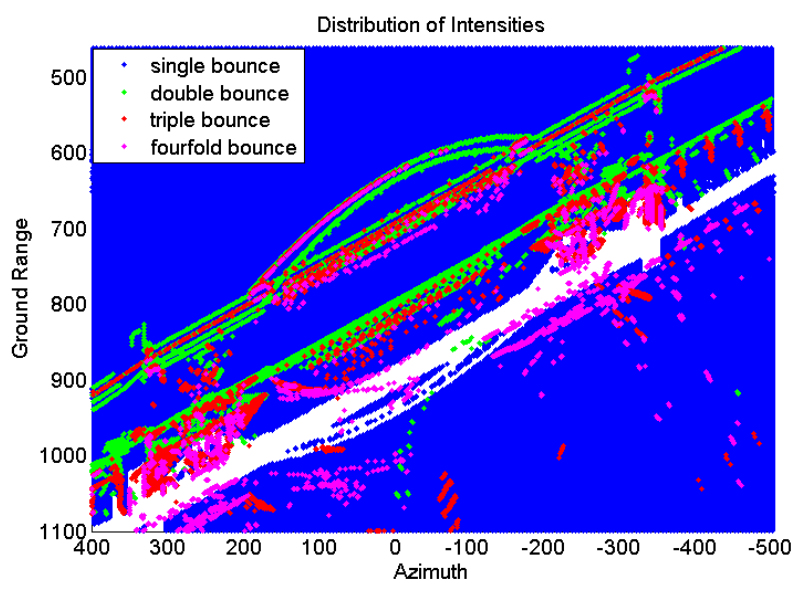

(b)

Figure 5. Simulated signal distribution of (a) ascending track (b) descending track. Blue: single bounce. Green: double bounce. Red: triple bounce. Magenta: fourfold bounce.

There are several points worth noting in Figure 5. First of all, the maximum bounce level is set to be four and the scene is assumed to be infinite so that each pixel has single bounce(blue color) backscatter. Secondly, the bounce level is closely related to the structure of the bridge and the relative position between the satellite and the model. Specifically, those points corresponding to double bounce(green color) well outline the main structure of the arc and road of the bridge. Illustrated in the bridge model coordinate as Figure 6, these double bounce mainly relate to the dihedral angle formed by the horizontal step/road and vertical railing. Meanwhile, those points corresponding to triple bounce(red color) only sparsely distributed in part of the near arc and road centre. Illustrated in the bridge model coordinate as Figure 7, the triple bounce points were surprised to locate in the similar position with double bounce. Taking the simulated reflectivity map of triple bounce into consideration, triple bounce is not the most significant signal contribution in this case and it's not specular reflection. Then, almost all of the fourfold bounce leads to ghost scatters. The extra propagation path results from the specular reflection on the water surface. The fake bridge on the bottom part of Figure 5 is identical to the bright fake bridge on the bottom part of Figure 4.Inevitably, there may be some offset as we lack the exact field-survey height of the bridge above the water surface. Besides, the elevation of water surface fluctuates with seasons. Finally, white color in Figure 5 represents the shadow of the bridge, which is also identical to Figure 4. 


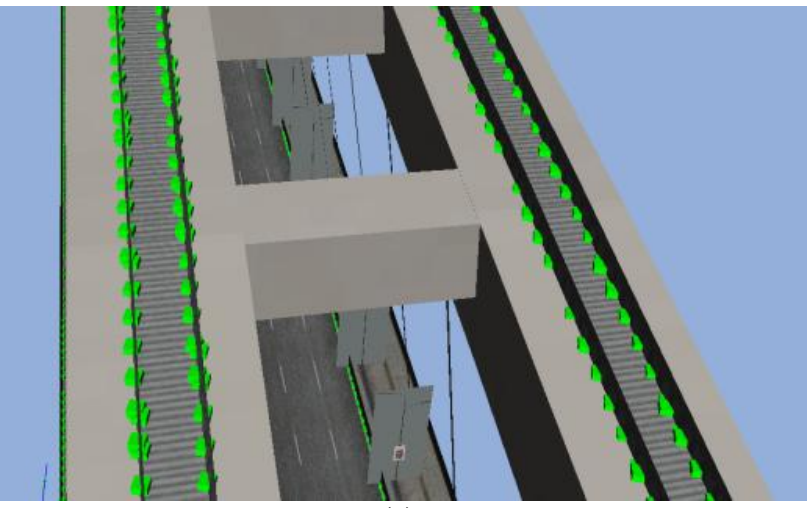

(a)

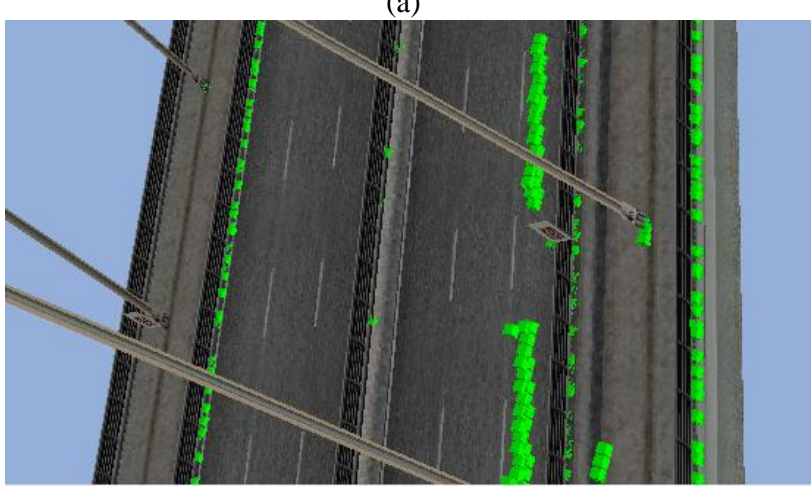

(b)

Figure 6. Double bounce(cubes in green color) on the (a) arc and (b) road of the Lupu Bridge. Note that there are sightseeing pavements on both sides of the arc and railings beside pavements and lanes.

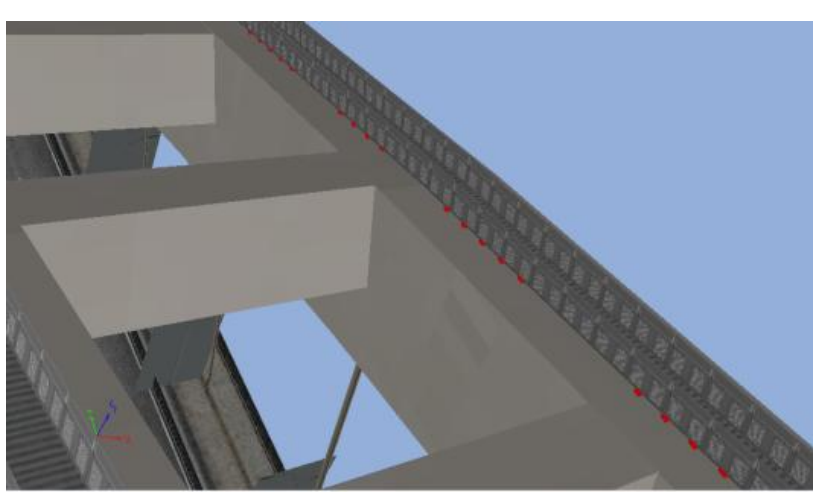

(a)

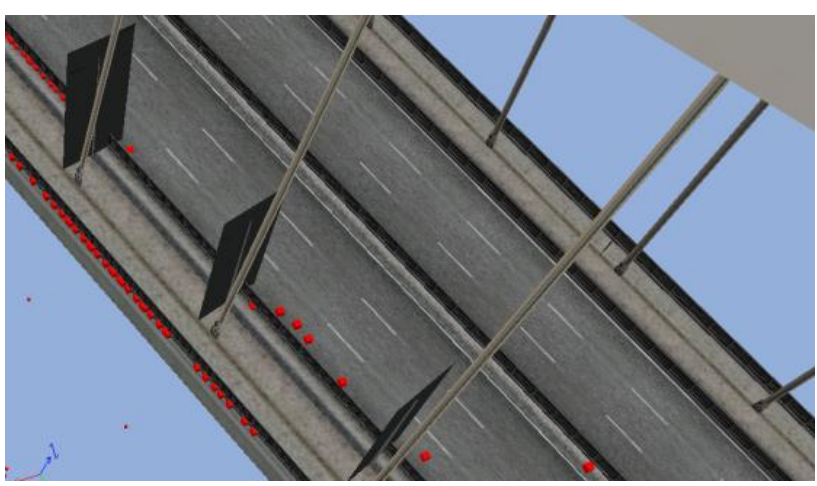

(b)

Figure 7. Triple bounce(cubes in red color) on the (a) arc and (b) road of the Lupu Bridge.

\section{STATISTIC INDEXES ANALYSIS OF RADAR SIGNAL}

There is no doubt that SAR image simulation approach is an appropriate way to recover the real situation in case of enough knowledge of the research area of interest. However, it's not practical to obtain the highly detailed object model in unfamiliar area by field survey as it's a laborious work and time-consuming. Thus we are looking for an alternative solution to multipath, namely, the product of the after-imaging, which refers to radar images. A SAR image consists of amplitude and phase. Both of them carry valuable information about the radar signal and illuminating object. For example, the SAR intensity image is a vivid representation of backscatter energy. A variety of patterns occurs with respect to the structure, orientation and surface material of illuminating object.

\subsection{SAR image processing}

In the following, the two tracks SAR images are processed with traditional PSInSAR method(Ferretti et al., 2001)by GAMMA software. As shown in Table 2, two methods are combined to select PS in GAMMA processing. The first one is based on the temporal variability of SLC intensity as point targets do not show the speckle behavior associated with distributed targets. This method can be related to two indexes: mean/sigma(standard deviation) ratio minimum threshold and intensity minimum threshold. The second method is based on the individual co-registered SLC. The PSs are selected based on their spectral characteristics such as coherence and mean/sigma ratio. Finally, 299 PSs in descending track and 600 PSs in ascending track are selected on the bridge.

\begin{tabular}{|l|c|c|}
\hline PS Selection Method & Index Name & Value \\
\hline Temporal Variability Of & MSR Minimum & 1.8 \\
\cline { 2 - 3 } SLC Intensity & PWR Minimum & 1.5 \\
\hline Spectral Characteristics & Coherence & $0.38-1$ \\
\cline { 2 - 3 } & MSR & $1.1-100$ \\
\hline
\end{tabular}

Table 2. PS selection methods used in image processing

\subsection{Statistic index analysis}

Later on, the statistic indexes of radar signal in time sequence such as the phase stability, mean amplitude, amplitude dispersion, coherence and mean-sigma ratio are summed and analyzed.

\subsubsection{Layover area}

In Figure 5(b), double bounce and triple bounce overlap on the upper left part of the outside bridge arc when compared to the PS in the image coordinate. To investigate the characteristic of layover case, the overlapped outside arc part is compared to its parallel inside arc as Table 3 and Figure 8 show.

In summary, statistic indexes of radar signal in time sequence such as the phase stability, coherence and mean/sigma ratio do not show much difference between layover case(double and triple bounce) and normal case, but indexes such as mean amplitude and amplitude dispersion vary dramatically. The outside arc whose amplitude results from both double and triple bounces is much brighter than the inside arc. Meanwhile, the outside arc has larger amplitude dispersion. This may be an indicator of the layover identification. 


\begin{tabular}{|l|c|c|c|}
\hline Index Name & & Outside Arc & Inside Arc \\
\hline Coherence & Minimum & 0.378 & 0.267 \\
\cline { 2 - 4 } & Maximum & 0.556 & 0.523 \\
\cline { 2 - 4 } & Mean & 0.445 & 0.413 \\
\hline \multirow{4}{*}{$\begin{array}{l}\text { Amplitude } \\
\text { Dispersion }\end{array}$} & Minimum & 4744.339 & 1811.445 \\
\cline { 2 - 4 } & Maximum & 16572.686 & 10169.064 \\
\cline { 2 - 4 } & Mean & 8814.163 & 6307.116 \\
\hline \multirow{3}{*}{$\begin{array}{l}\text { Mean/Sigma } \\
\text { Ratio }\end{array}$} & Minimum & 1.118 & 1.024 \\
\cline { 2 - 4 } & Maximum & 1.229 & 1.252 \\
\cline { 2 - 4 } & Mean & 1.178 & 1.160 \\
\hline Phase & Minimum & 1.619 & 1.731 \\
\cline { 2 - 4 } & Maximum & 1.971 & 2.038 \\
\cline { 2 - 4 } & Mean & 1.830 & 1.859 \\
\hline Mean \\
Amplitude & Minimum & 5868.171 & 2578.514 \\
\cline { 2 - 4 } & Maximum & 22477.543 & 13791.200 \\
\cline { 2 - 4 } & Mean & 10609.032 & 7199.326 \\
\hline
\end{tabular}

Table 3. Statistic indexes of radar signal in time sequence corresponding to part of two parallel arcs (Note that the amplitude has not been normalized)

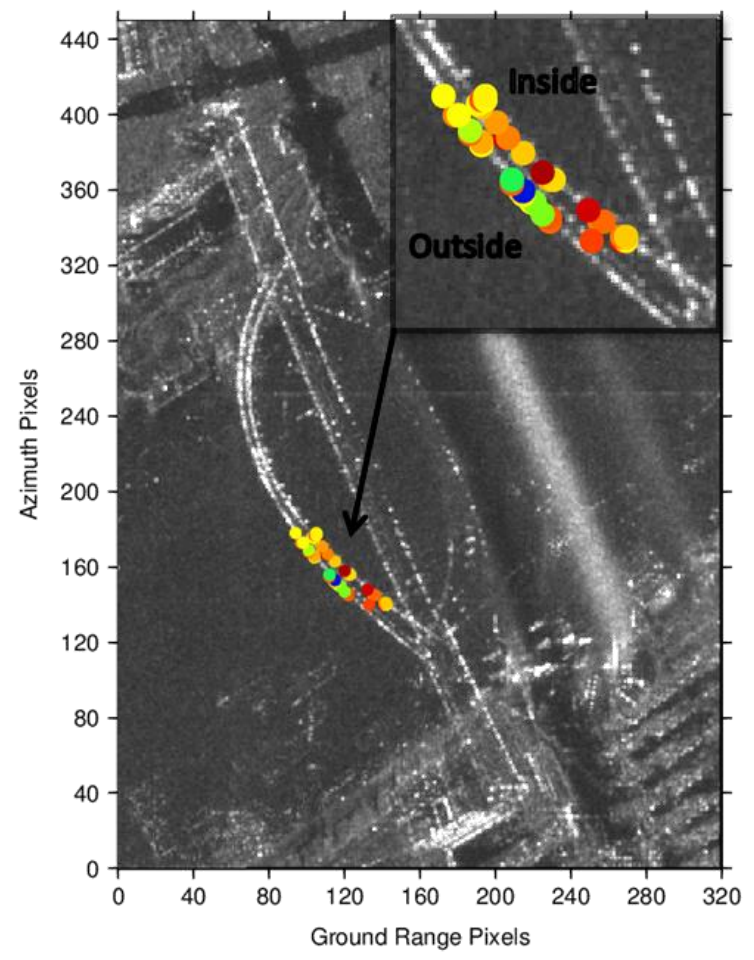

$3000 \quad 6000 \quad 9000 \quad 12000 \quad 15000$

Figure 8. Amplitude dispersion of part bridge arc (Note that the amplitude has not been normalized)

\subsubsection{Ghost scatter}

Multipath may lead to ghost scatters such as the reflection of the bridge in the water. This kind of ghost scatters are normally far from their real location but keep the same pattern. To validate the ghost scatter recognition result, coordinate transformation between SAR image simulation approach and SAR statistic index analysis is necessary. One way is to link RaySAR signal contribution coordinate(Figure 5) with PS in SAR image coordinate(Figure 8), another way is to link the RaySAR signal contribution in 3D model coordinate with 3D PS location. Limited by the time, this work will be done in the future.

\section{CONCLUSION AND OUTLOOK}

In the Lupu Bridge case, SAR image simulation method based on RaySAR has been used for multipath analysis at first. A basic knowledge about the backscatter energy strength and multipath location of each bounce level is given by reflectivity map and signal distribution map generated from RaySAR. Simulated SAR image signatures of bounce levels higher than two have been projected back to the $3 \mathrm{D}$ model coordinate and linked to the bridge structure. The simulation results confirm that ghost corners are crucial for the occurrence of multipath effects. For bridges above flat water surface, specular reflection including the water should cause enough attention.

Expect for the SAR simulation approach, statistic indexes of the SAR image could be a powerful complement for multipath and layover pixel recognition. As found in our study, layover pixels have much larger mean amplitude and amplitude dispersion than normal pixels.

In future work, coordinate transformation between (a)RaySAR reflectivity maps and SAR averaged intensity images (b)RaySAR signal distribution maps and PSs' position is necessary to validate the multipath recognition result. Besides, certain algorithm related to statistic indexes should be proposed to better link the multipath pixels with their special statistical features.

\section{ACKNOWLEDGEMENTS}

The 3D model of the Lupu Bridge was downloaded from Google 3D warehouse and many thanks to GAMMA and RaySAR software. This research was supported by the State Key Development Program for Basic Research of China (No.2013CB733304). Thanks DLR Italian Space Agency for COSMO-SkyMed images

\section{REFERENCES}

Auer, S., Gernhardt, S. and Bamler, R., 2011. Ghost persistent scatterers related to multiple signal reflections. IEEE Geosci. Remote Sens.Letters, IEEE 8 (5), 919-923.

Auer, S. J., 2011. 3D synthetic aperture radar simulation for interpreting complex urban reflection scenariosl. Ph.D. Dissertation of Technical University of Munich.

Balz, T. and Stilla, U., 2009. Hybrid GPU-Based Single- and Double-Bounce SAR Simulation. IEEE Trans. Geosci. Remote Sens. 47 (10), 3519-3529.

Ferretti, A., Prati, C. and Rocca, F., 2001. Permanent scatterers in SAR interferometry. IEEE Trans. Geosci. Remote Sens. 39 (1), 8-20.

Hammer, H. and Schulz, K., 2009. Coherent simulation of SAR images. Proc SPIE ImageSignal Process. Remote Sens. XV 7477, pp. 74771K-1-8. 